Article

\title{
Full-Duplex NOMA Transmission with Single-Antenna Buffer-Aided Relays
}

\author{
Nikolaos Nomikos $1, * \mathbb{(})$, Panagiotis Trakadas ${ }^{2}\left(\mathbb{D}\right.$, Antonios Hatziefremidis $^{2}(\mathbb{D}$ ) and \\ Stamatis Voliotis ${ }^{2}$ (1) \\ 1 Department of Information and Communication Systems Engineering, University of the Aegean, \\ 83200 Samos, Greece \\ 2 General Department, National and Kapodistrian University of Athens, 34400 Psahna, Greece; \\ ptrakadas@uoa.gr (P.T.); ahatzie@uoa.gr (A.H.); svoliotis@uoa.gr (S.V.) \\ * Correspondence: nnomikos@aegean.gr
}

Received: 11 November 2019; Accepted: 3 December 2019; Published: 4 December 2019

\begin{abstract}
The efficient deployment of fifth generation and beyond networks relies upon the seamless combination of recently introduced transmission techniques. Furthermore, as multiple network nodes exist in dense wireless topologies, low-complexity implementation should be promoted. In this work, several wireless communication techniques are considered for improving the sum-rate performance of cooperative relaying non-orthogonal multiple access (NOMA) networks. For this purpose, an opportunistic relay selection algorithm is developed, employing single-antenna relays to achieve full-duplex operation by adopting the successive relaying technique. In addition, as relays are equipped with buffers, flexible half-duplex transmission can be performed when packets reside in the buffers. The proposed buffer-aided and successive single-antenna (BASSA-NOMA) algorithm is presented in detail and its operation and practical implementation aspects are thoroughly analyzed. Comparisons with other relevant algorithms illustrate significant performance gains when BASSA-NOMA is employed without incurring high implementation complexity.
\end{abstract}

Keywords: NOMA; full duplex; successive relaying; buffer-aided relaying

\section{Introduction}

Mobile data traffic has been increasing at a dramatic pace due to the emergence of Internet-of-Things (IoT) applications [1], resulting in coexisting human and machine traffic. So, novel techniques are required to provide improved spectral efficiency and transmission reliability. Regarding spectral-efficient communication, non-orthogonal multiple access (NOMA) has been proposed as a promising solution to overcome the inefficiency of orthogonal resource allocation in multi-user networks [2]. In contrast to orthogonal multiple access (OMA), NOMA enables multiple devices to be served on the same resources in the power domain (PD) or code domain (CD) [3]. The PD NOMA paradigm relies on combining superposition coding at the transmitters and successive interference cancellation (SIC) at the receivers and its performance depends on various parameters, such as user channel asymmetry, rate requirements and cooperation among devices [4,5]. Moreover, in order to strengthen the reliability of fifth generation (5G) communication, buffer-aided (BA) cooperative relaying has the potential to improve the wireless conditions, offering increased diversity and enhancing the outage and throughput performance, as shown in various studies [6,7]. The use of buffers enables hybrid transmission modes, due to the decoupling of the reception and transmission phases, as relays might have packets to forward even if reception was not possible in the previous phase. The survey in [7] presented various opportunistic relay selection (ORS) algorithms comprising half-duplex (HD), full-duplex (FD) and successive relaying (SuR), showing significant outage and 
throughput performance gains. In addition, low latency is a prerequisite for highly demanding applications, such as augmented/virtual reality, public safety applications and ultra-high definition teleconferencing among others and thus apart from FD transmission, delay-awareness must be integrated in cooperative relaying $[8,9]$.

\subsection{Background}

BA relays were initially proposed in HD networks with single-antenna relays. These networks suffer from half-duplex rate losses, as relays are not able to receive and transmit at the same time. Firstly, the paper in [10] suggested to equip relays with buffers in two-hop ORS topologies. In greater detail, each time-frame was divided to two time slots, with each one allocated to the source-relay $(\{S \rightarrow R\})$ and relay-destination $(\{R \rightarrow D\})$ transmissions, forming the hybrid relay selection (HRS). Further improvements were provided by max - link, an ORS algorithm that was proposed in [11], achieving increased diversity due to BA relays and flexible selection of the strongest link among all the available ones. The performance analysis showed that the use of max - link offers a diversity gain of $2 K$ when $K$ relays and large buffers are available in the network. Focusing on single relay networks with multiple antennas, the authors in [12], analyzed the performance of BA relaying with adaptive link selection, concluding that the performance of HD relaying can surpass that of ideal FD relaying, given that the number of antennas at the source and the destination is greater than the number of available antennas at the relay. More recently, further diversity gains were introduced in BA relay networks through broadcast transmissions in the $\{S \rightarrow R\}$ links [13], resulting in beneficial packet redundancy, while the work in [14] proposed low-complexity distributed solutions to mitigate the impact of various issues, such as errors in the feedback channel and relay selection using outdated channel state information (CSI).

Without departing from single-antenna relay networks, several studies aimed at overcoming their inherent half-duplex constraint and provided throughput gains. The paper in [15] introduced hybrid BA ORS, merging HD relaying and SuR in a topology with isolated relays where the inter-relay interference (IRI), arising from the successive source and relay transmissions, was considered negligible. Both adaptive and fixed-rate transmissions were studied, revealing that space full-duplex (SFD) max-max relay selection (MMRS) can double the capacity of HD schemes in the former case, and offer coding gain, as well as diversity gain equal to the number of relays, in the latter case. Then, the degrading effect of IRI and its mitigation through proper relay-pair selection and interference cancellation were investigated in [16], while transmit power adaptation in fixed-rate communication and the integration of max - link and SuR was proposed in [17]. Comparisons with standalone HD and FD solutions indicated significant resiliency against outages and increased throughput. Another improvement towards low-delay SuR was given in [18]. The proposed low-latency for the successive opportunistic relaying (LoLA4SOR) algorithm considered the buffer length in both $\{S \rightarrow R\}$ and $\{R \rightarrow D\}$ links and, if SuR failed, delay-aware (DA) HD relaying was performed, exhibiting reduced delay and robustness against outages. Furthermore, optimal scheduling towards delay-awareness and IRI mitigation based on either dirty paper coding or SIC for topologies with two relays, was examined in [19]. More recently, apart from data buffers, energy buffers storing the amount of energy that is harvested from wireless transmissions have been incorporated in BA SuR networks with single [20] or multiple relays [21], enabling network lifetime prolongation.

As spectrum scarcity threatens the operation of current wireless networks, comprising humans and machines, spectral efficient transmission is of vital importance. In this research field, NOMA has emerged as a promising technique and its integration in cooperative relaying networks has received various contributions. The work in [22] assigned users with better channel conditions to decode the messages of weak users, relaying them in the next time slot, thus promoting user fairness through homogeneous cell coverage. Then, ORS was studied in [23], introducing a two-stage relay selection, increasing the diversity gain, compared to conventional max-min ORS. Another ORS algorithm for topologies where users are employed to serve as relays was given in [24], resulting in coverage and 
capacity enhancement. More specifically, a range-division user relay selection scheme was devised, dividing the circular cell in continuous annular areas and performing ORS in each one. Other works, targeted the sum-rate of two-hop NOMA relay networks, jointly studying NOMA and SuR $[25,26]$. The proposed solutions created virtual FD relay networks by employing two dedicated relays to perform SuR and serve two users through NOMA. Likewise, a four-user topology where two users were chosen to perform SuR and serve the remaining two users using NOMA was shown in [27], achieving significant sum-rate gains.

Apart from employing SuR to overcome sum-rate losses, the use of buffers can also improve the performance of NOMA in HD relay networks. An adaptive mode selection, enabled through a single BA relay, was given in [28]. So, switching between NOMA and OMA when the former was infeasible, allowed a sum-rate increase, compared to OMA-based BA relaying and NOMA relaying without buffers. In cases when CSI at the transmitter might not be available, the authors in [29] presented adaptive link selection, deriving the outage probability expressions and showing a diversity gain of two for buffer sizes larger than two packets. In networks where multiple BA relays are present, two BA-ORS algorithms were given in [30], choosing the best relay, by considering the buffer state information (BSI) and adopting $\{R \rightarrow D\}$ prioritization for reduced latency. Then, the potential of BA-ORS in NOMA and hybrid NOMA/OMA networks was presented in [31,32], where hybrid NOMA/OMA selection policies were devised, further improving the sum-rate performance in the downlink and the uplink of two-hop networks, respectively.

\subsection{Contributions}

It is evident that significant advances have been made in NOMA-based cooperative relay networks, regarding the sum-rate performance, even in HD networks consisting of single-antenna relays and users. Still, the potential of SuR using BA relays in NOMA networks has not been examined and thus this paper aims to fill this gap. More specifically,

- A novel BA ORS algorithm is presented for two-hop NOMA networks, called buffer-aided successive single-antenna (BASSA) NOMA, targeting to provide FD relaying operation by relying only on low-complexity single antenna relays.

- Several wireless communication techniques are presented and integrated in BASSA-NOMA, such as successive broadcasting in the $\{S \rightarrow R\}$ links and $\{R \rightarrow D\}$ transmission through a selected relay, thus leading to FD operation with IRI, while guaranteeing that $\{R \rightarrow D\}$ transmission considers the selected transmitting relay's BSI, avoiding instances of storing packets for many time slots and thus increased delay.

- Furthermore, the steps of BASSA-NOMA are given in detail and various practical issues are discussed, such as power allocation and low-complexity network coordination for choosing the best decoding strategy at the destinations.

- Performance comparisons with other BA NOMA solutions show that even though BASSA-NOMA does not incur high implementation complexity, in terms of CSI acquisition and processing, it enhances the sum-rate and delay performance of the network, while maintaining reduced outages.

Overall, BASSA-NOMA represents an attractive low-complexity solution for two-hop NOMA networks with the potential to be adopted in heterogeneous contexts where relay nodes might not always posses high hardware and processing capabilities, such as in networks where users [33], vehicles [34] and drones [35] assume the role of relays.

\subsection{Structure}

The paper is organized as follows. In Section 2, the system model is given, while Section 3 presents relevant relay selection algorithms. Next, Section 4, presents BASSA-NOMA and then Section 5 includes comparisons between the proposed, as well as relevant schemes. Finally, Section 6 includes conclusions and possible future directions. 


\section{System Model}

This work studies a two-hop cooperative relay network, comprising one source $S$, two destinations $D_{1}$ and $D_{2}$ and a cluster $\mathcal{C}$ with $K$ decode-and-forward (DF) relays $R_{k} \in \mathcal{C}(1 \leq k \leq K)$. DF avoids the detrimental effect of noise amplification by the relays, at the cost of higher implementation complexity, compared to amplify-and-forward relays, as it has been shown in various works on cooperative relaying in multi-user networks [36,37]. At the same time, relays are clustered in such a way, so that communication among them can be reliably established in order to ensure error-free signaling and coordination. Towards this end, the relays are positioned relatively close together, according to location-based clustering and after a long-term routing process, variations due to pathloss and shadowing effects are tracked. It should be noted that relay clustering is often assumed in the literature, as, e.g., in [16,38]. Each node is equipped with a single-antenna and relays operate in HD mode where concurrent signal transmission and reception is not possible. Furthermore, due to excessive fading conditions in both source-destination $(\{S \rightarrow D\})$ links, end-to-end communication is only established via the two-hop relay links. Each relay $R_{k}$ has a buffer to store a maximum number $L_{k}$ of successfully decoded packets in order to forward them at a later instance to the destinations. All buffers are of equal length, i.e., $L_{k}=L, \forall k \in\{1,2, \ldots, K\}$ while the vector including the queue sizes at the buffers of all relays is denoted by $Q \triangleq\left(Q_{1}, Q_{2}, \ldots, Q_{K}\right)$. Initially, each relay buffer has $Q_{k}$ packets, while some buffers might be empty (i.e., $Q_{k}=0$ for an arbitrary $k$ ). Figure 1 depicts an instance of this BA relay-assisted NOMA network, where the source performs broadcasting towards $K-1$ relays, while a selected transmitting relay forwards previously received packets to the two destinations, leveraging successive source and relay transmissions for FD NOMA operation.

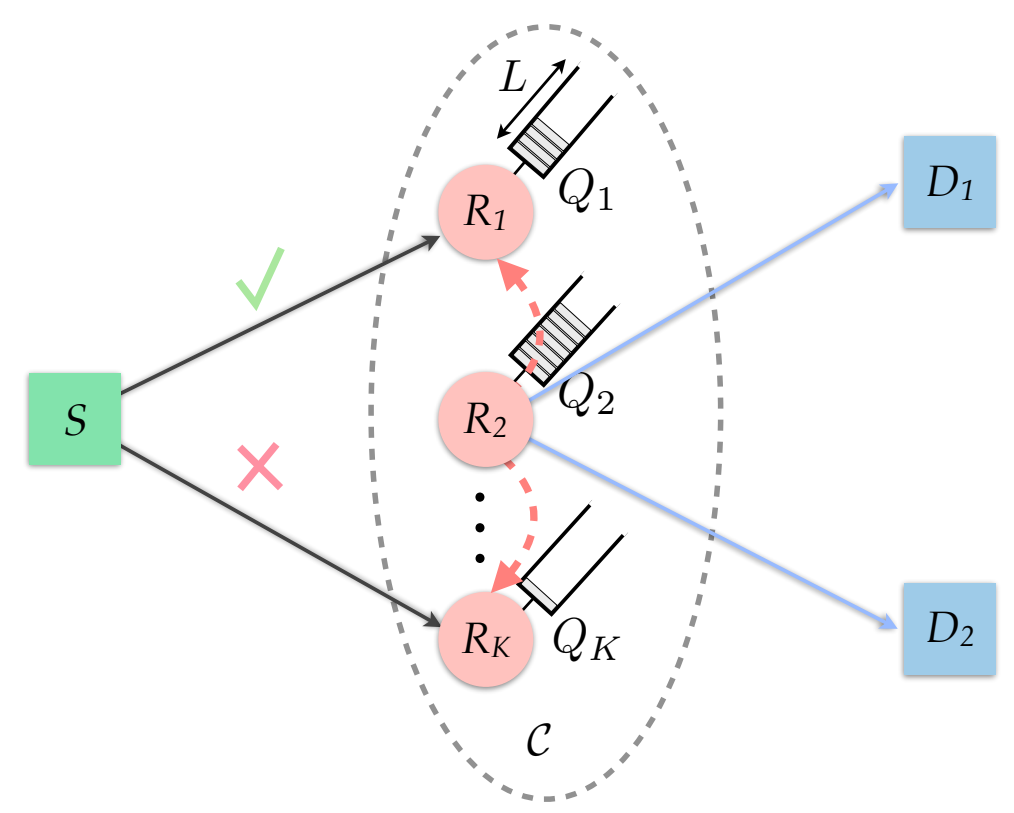

Figure 1. Source $S$ communicates with two destinations $D_{j}, j \in\{1,2\}$ through a cluster of relays $R_{k} \in \mathcal{C}, k \in\{1, \ldots, K\}$.

The transmission is divided in time slots and at each one simultaneous source and relay transmissions are allowed, using transmit power values $P_{S}$ and $P_{R_{k}}$, respectively. A saturated source having always data for transmission, using an information rate of $r_{0} \mathrm{bps} / \mathrm{Hz}$ is assumed. The retransmission process is based on an acknowledgment/negative-acknowledgment (ACK/NACK) mechanism, employing the receivers (the relays and/or the destination) to broadcast short-length error-free packets over a separate narrow-band channel, notifying the network on whether or not, a specific packet was successfully received. Additionally, it is considered that the receivers are able to accurately estimate the CSI, while the relays have full CSI knowledge of the $\{R \rightarrow D\}$ links. As 
buffering at the relays is available, it is possible for the selected transmitting relay to forward a packet to the two destinations that was received in a time slot that is different from the preceding one. As a result, the destinations might have to reorder the received packets, in order to correctly decode the desired information, a necessary process in all BA relaying protocols. Still, this can be easily achieved at a low-complexity by including a sequence number in each packet.

Regarding the wireless channel conditions, it is assumed that channel quality is degraded by additive white Gaussian noise (AWGN) and for simplicity, the power of the AWGN is assumed to be normalized with zero mean and unit variance. The fading environment is modelled as frequency flat Rayleigh block fading, unless otherwise stated, following a complex Gaussian distribution with zero mean and variance $\sigma_{i j}^{2}$ for the $i$ to $j$ link. Moreover, the channel gains are $g_{i j} \triangleq\left|h_{i j}\right|^{2}$ and exponentially distributed.

Since successive source broadcasting and relay transmissions are performed in the network, all $K$ relays are active at each time slot, as $K-1$ relays attempt to receive the source signal, while a selected transmitting relay is forwarding a previously received packet to the two destinations. Thus, the HD rate loss of single-antenna relays can be alleviated through SuR. This virtual FD operation enables the destination to receive one packet per time slot, excluding the first one. Still, as a direct result of the successive transmissions, IRI arises and an efficient selection algorithm has to take into consideration its impact on the $K-1$ relays' reception. In case SuR fails, a complete network outage can be avoided by incorporating a low-complexity mechanism, allowing at least an HD transmission towards the relays or the destinations.

\section{Relevant Algorithms}

In this section, relevant relay selection algorithms are given in detail, illustrating their main characteristics upon which, BASSA-NOMA is formulated.

\subsection{Delay-Aware Max-Link: Adaptive Link Selection, BSI Consideration and $\{R \rightarrow D\}$ Prioritization}

Initial attempts at providing HD BA ORS algorithms relied on fixed scheduling for the source and relay transmissions. For the first time, this limitation was relaxed in [11], enabling the flexible allocation of each time slot to either an $\{S \rightarrow R\}$ or $\{R \rightarrow D\}$ transmission, according to the instantaneous CSI and the status of the relays' buffers, activating the strongest link between all the available ones. Still, max - link did not integrate any mechanism to offer delay-awareness and so, further extensions were given in $[9,39]$. It was shown that the average packet delay can be reduced by prioritizing the $\{R \rightarrow D\}$ transmission, prompting packets to leave sooner from the buffers. The DA - max - link algorithm operates as follows:

1. In an arbitrary time slot, from the set $\mathcal{F}_{R D}$, consisting of relays that are able to perform an $\{R \rightarrow D\}$ transmission, DA - max - link considers the number of packets residing in the buffer of each relay. So, the relay having the maximum number of packets is activated to perform a transmission in the $\{R \rightarrow D\}$ link.

2. In case an $\{R \rightarrow D\}$ transmission cannot be performed, DA - max - link aims at activating the relay that can perform an $\{S \rightarrow R\}$ reception, having the minimum buffer length. Nonetheless, if no feasible $\{S \rightarrow R\}$ link exists, a network outage occurs, since packet transmission cannot be performed.

DA - max - link avoids cases of buffer overflow and underflow by integrating BSI into the relay selection process. If more than one relays have equal buffer sizes, a relay is randomly selected among them. Moreover, due to $\{R \rightarrow D\}$ prioritization, the average delay performance improves, thus being an attractive solution for delay-sensitive applications. 


\subsection{LoCo-Link: Source Broadcasting, BSI Consideration and $\{R \rightarrow D\}$ Prioritization}

The low-complexity (LoCo - Link) selection algorithm was introduced in [14], targeting to improve the average delay performance of BA HD relaying, at a low implementation complexity, in terms of CSI requirements. LoCo - Link's operation is described below:

1. In contrast to max - link, where the strongest link was selected between the $2 K$ available ones in a $K$ relay network, LoCo - Link firstly focuses on the $\{R \rightarrow D\}$ links, selecting in each time slot, the $\{R \rightarrow D\}$ link belonging in $\mathcal{F}_{R D}$, having the maximum number of packets in its buffer. If multiple relays have the same buffer length, one of them is randomly selected.

2. If $\mathcal{F}_{R D}$ is empty, as a result of severe fading or empty buffers, the source broadcasts its packets to all the relays. Thus, there might exist more than one relays forming the set $\mathcal{F}_{S R}$, including the feasible $\{S \rightarrow R\}$ links, being able to successfully receive the source's packets. It is evident that in subsequent time slots, the diversity of $\{R \rightarrow D\}$ transmissions increases, compared to schemes with unicast $\{S \rightarrow R\}$ transmissions, such as DA - max - link.

LoCo - Link provides several gains to the two-hop BA networks, reducing the average end-to-end delay without degrading the diversity of the network. In addition, when a distributed implementation is adopted, the issue of relay selection with outdated CSI can be fully mitigated.

\subsection{LoLa4SOR: Hybrid Successive and Half-Duplex Relaying with BSI Consideration}

Various DA BA algorithms have adopted $\{R \rightarrow D\}$ prioritization to avoid buffers storing a large number of packets $[9,39]$. Still, it has been observed that although reduced latency was achieved, diversity was compromised due to instances of empty buffers. In order to safeguard the diversity of the transmission while offering increased rate through SuR, LoLa4SOR was proposed in [18], based on the hybrid combination of SuR and HD relaying. LoLa4SOR operates as follows:

1. The possibility for SuR is examined, examining possible relay pairs while considering the inter-relay CSI, at each time slot. At the same time, low-latency dictates that buffer length should be kept small without ignoring the diversity. So, relays having the largest buffer lengths and relays with the smallest ones should be prioritized as transmitting and receiving relays, respectively. Then, by denoting as $\mathcal{F}_{\text {pairs }}$ the set of relay pairs that can perform SuR, at the start of each time slot, a relay-pair $\left(R_{i}, R_{j}\right)$ in links $\left\{S \rightarrow R_{i}, R_{j} \rightarrow D\right\}$, among all the feasible ones for SuR, i.e., $\left(R_{i}, R_{j}\right) \in \mathcal{F}_{\text {pairs }}$, is activated as:

$$
\left(R_{i^{\star}}, R_{j^{\star}}\right)=\arg \max _{\left(R_{i}, R_{j}\right) \in \mathcal{F}_{\text {pairs }}}\left\{\left(L-Q_{i}\right)^{2}+Q_{j}^{2}\right\}
$$

where $\left(R_{i^{\star}}, R_{j^{\star}}\right)$ denote the optimal relay-pair $\left(R_{i}, R_{j}\right)$, i.e., the one providing the maximum utility in Equation (1). If two or more relay pairs have the same utility, then diversity is prioritized, and from the set of relay pairs with the maximum utility, denoted by $\mathcal{F}_{\text {pairs }}^{\star}$, the one with the maximum $\{S \rightarrow R\}$ link utility is activated, i.e.,

$$
\left(R_{i^{0}}, R_{j^{0}}\right)=\arg \max _{\left(R_{i^{\star}}, R_{j^{\star}}\right) \in \mathcal{F}_{\text {pairs }}^{\star}}\left\{\left(L-Q_{i}\right)^{2}\right\} .
$$

If two or more relay pairs still provide the same maximum utility, denoted by $\mathcal{F}_{\text {pairs }}^{o}$ one of them is randomly selected.

2. In cases where SuR is not possible, the efficient delay- and diversity-aware max - link (DDA - max - link) HD algorithm, introduced in [9] is activated. DDA - max - link avoids the selection of relays with buffers being on the brink of underflow or overflow by setting appropriate thresholds and activating such relays only to avoid a network outage. 


\subsection{BA-NOMA/OMA: Flexible Multiple Access with Optimal Power Allocation}

In downlink NOMA networks with a single source, multiple BA relays and two destinations, hybrid BA-NOMA/OMA was proposed in [31], integrating broadcasting in the $\{S \rightarrow R\}$ link and exploiting BSI in the $\{R \rightarrow D\}$ NOMA transmission. The steps of BA-NOMA/OMA are given in the following paragraphs:

1. In the $\{S \rightarrow R\}$ link, BA-NOMA/OMA enables the source to broadcast a combined packet containing the information of both destinations with rate $r_{1}+r_{2}$, towards all the relays. Then, each relay uses the CSI of the $\{R \rightarrow D\}$ links to examine if PD NOMA is possible through the transmission of a superposition-coded packet to the two destinations. Still, NOMA might not be possible, i.e., due to low transmit signal-to-noise ratio (SNR), insufficient channel asymmetry between the destinations or high rate demands. So in order to avoid a complete network outage, OMA is performed, investigating if at least a single destination $D_{j}, j \in\{1,2\}$ can receive a packet from the relays included in $\mathcal{F}_{R D_{j}}$, representing the set of feasible OMA links towards $D_{j}$.

2. When OMA is activated, user fairness is guaranteed since at odd time slots, first, $D_{1}$ is examined if it can receive its packet from $R_{k} \in \mathcal{F}_{R D_{1}}$, else $D_{2}$ is examined if it can receive its packet from $R_{k} \in \mathcal{F}_{R D_{2}}$. Similarly, at even time slots this process is reversed. When relays with equal buffer lengths exist, random selection occurs in the $\{R \rightarrow D\}$ links.

BA-NOMA/OMA is an efficient relay selection algorithm, combining the benefits of both multiple-access schemes, thus improving the average sum-rate performance of multi-user two-hop HD relay networks.

\section{The BASSA-NOMA Algorithm}

\subsection{Algorithmic Description}

BASSA-NOMA targets to improve the sum-rate performance of two-hop NOMA cooperative relay networks, relying only on single-antenna relays with low-complexity. So, various transmission techniques are integrated, including successive source broadcasting and relay transmissions, as well as buffering at the relays. The procedure followed by each relay $R_{k}$, under the BASSA-NOMA algorithm, in each time slot, is given in Algorithm 1.

More specifically, firstly, BASSA-NOMA aims at selecting a transmitting relay $R_{k}$ to simultaneously serve $D_{1}$ and $D_{2}$ using PD NOMA. Relay selection relies on the availability of both $\{R \rightarrow D\}$ links' CSI, determining whether or not a NOMA transmission can be successfully performed, as well as each relay's BSI, deciding which one has the maximum number of packets in its buffer (line 1). So, each candidate transmitting relay $R_{k}$ appropriately chooses the power allocation coefficient, according to its respective $\{R \rightarrow D\}$ CSI (line 2). Simultaneously and in a distributed manner, each candidate relay $R_{k}$ sets its timer to be inversely proportional to the number of packets $Q_{k}$, residing in its buffer (lines 3 and 4). In case a transmitting relay is selected (line 5), $\{S \rightarrow R\}$ broadcasting and $\{R \rightarrow D\}$ transmission are concurrently performed and thus the remaining $K-1$ relays attempt to receive the source's broadcast signal, containing the information signals of $D_{1}$ and $D_{2}$ with rate $r=r_{1}+r_{2}$, while subjecting to IRI (lines 6 and 7). Through broadcasting, more packets are available at the relays' buffers and more importantly, CSI at the source is not required, thus significantly reducing the implementation complexity. If, on the other hand, a transmitting relay was not selected, all $K$ relays are available to listen to the source's broadcast transmission, as long as they have space in their buffers (line 10). Finally, when the two destinations receive packets from the transmitting relay, ACKs, containing the relevant packets IDs are broadcasted to all the relays, prompting them to discard these packets from their buffers (line 12). The adoption of BASSA-NOMA enables the network to operate in FD mode without depending on relays with multiple antennas, nor on advanced self-interference cancellation schemes, as it is usually the case in two-hop networks, where simultaneous transmission and reception take place through the same relay. 


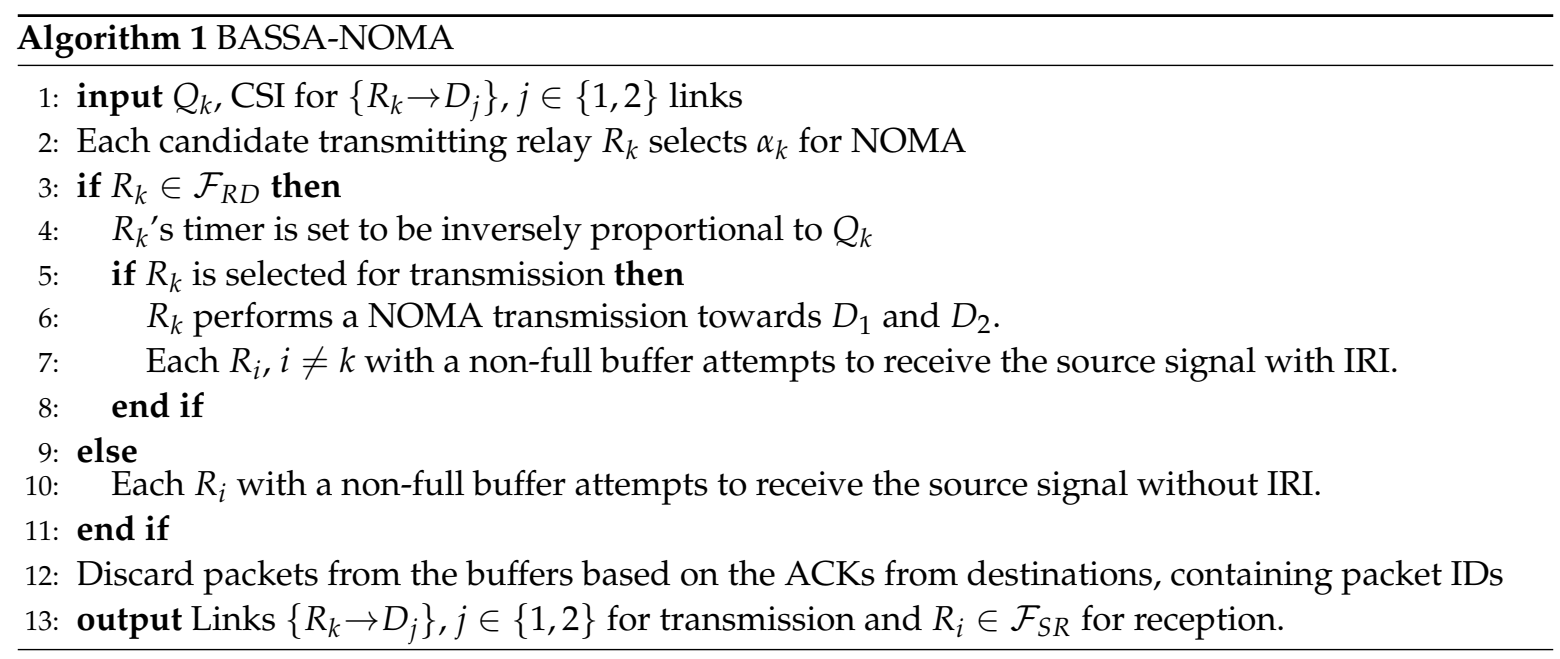

As at an arbitrary time slot, BASSA-NOMA initially checks the $\{R \rightarrow D\}$ links on whether or not a NOMA transmission is possible, firstly, details on the $\{R \rightarrow D\}$ transmission are given.

\subsection{Transmission in the $\{R \rightarrow D\}$ Link}

When a transmitting relay $R_{k}$ is selected, the information symbols of $D_{1}$ and $D_{2}$ are superimposed following the PD NOMA paradigm. In greater detail, the transmitted superimposed information symbol, containing the information symbols $x_{1}$ and $x_{2}$ of each destination, is described as:

$$
x=\sqrt{\alpha_{k}} x_{1}+\sqrt{1-\alpha_{k}} x_{2}
$$

with $\mathbb{E}\left[\left|x_{1}^{2}\right|\right]=\mathbb{E}\left[\left|x_{2}^{2}\right|\right]=1$ and $0 \leq \alpha_{k} \leq 1$.

Then, $D_{1}$ will receive an information symbol $y_{1}$ containing the desired symbol, as well as the symbol of $D_{2}$, i.e.,

$$
y_{1}=h_{R_{k} D_{1}} \sqrt{\alpha_{k} P_{R_{k}}} x_{1}+h_{R_{k} D_{1}} \sqrt{\left(1-\alpha_{k}\right) P_{R_{k}}} x_{2}+\eta_{1} ;
$$

correspondingly, the received information symbol $y_{2}$ at $D_{2}$ is given by:

$$
y_{2}=h_{R_{k} D_{2}} \sqrt{\alpha_{k} P_{R_{k}}} x_{1}+h_{R_{k} D_{2}} \sqrt{\left(1-\alpha_{k}\right) P_{R_{k}}} x_{2}+\eta_{2},
$$

where $\eta_{1}$ and $\eta_{2}$ denote the AWGN at each destination.

An important parameter that must be optimally determined is $\alpha_{k}$ representing the power allocation of the transmitting relay $R_{k}$ to the superimposed signals. Here, the relays poses full CSI of the $\{R \rightarrow D\}$ links and power allocation can be dynamically adjusted in each time slot. The value of $\alpha_{k}$ is determined in order to increase the chances of successful SIC to decode $x_{1}$ and $x_{2}$. Thus, in order to achieve SIC, the signal-to-interference-plus-noise ratio (SINR), for at least one of the symbols, should be greater than or equal to a threshold at both destinations. This process is followed by all the relays except from the possible transmitting one, leading to the formation of the set of relays that can perform $\{R \rightarrow D\}$ NOMA transmissions.

As an illustrative example, the decoding of $x_{2}$ at both destinations is performed as follows:

$$
\Gamma_{R_{k} D_{j}}\left(P_{R_{k}}\right)=\frac{\left(1-\alpha_{k}\right) P_{R_{k}} g_{R_{k} D_{j}}}{\alpha_{k} P_{R_{k}} g_{R_{k} D_{j}}+\sigma_{D_{j}}^{2}} \geq \gamma_{j}, \quad j \in\{1,2\} .
$$


Note that $\gamma_{j} \equiv 2^{r_{j}}-1$. Then, once $x_{2}$ is successfully decoded and subtracted, $x_{1}$ can be decoded interference-free at $D_{1}$ according to:

$$
\Gamma_{R_{k} D_{1}}\left(P_{R_{k}}\right)=\frac{\alpha_{k} P_{R_{k}} g_{R_{k} D_{1}}}{\sigma_{D_{1}}^{2}} \geq \gamma_{1}
$$

There are different methods of determining the power allocation coefficient $\alpha_{k}$ in NOMA networks. In this work, power allocation follows the method that was developed in [31] which considered a two-hop relay network with two destinations with possibly heterogeneous rate requirements, as it is the case of coexisting IoT devices and users. Since a similar $\{R \rightarrow D\}$ link setting is studied here, readers are referred to that work for more details on calculating $\alpha_{k}$.

The outage probability of NOMA in the $\{R \rightarrow D\}$ link is equal to:

$$
p_{\text {out }\{R \rightarrow D\}}=\mathbb{P}\left[\alpha_{k, \min }>\min \left\{1, \alpha_{k, \max }\right\}\right] .
$$

where $\alpha_{k, \min }$ and $\alpha_{k, \max }$ are expressed as [31]:

$$
\begin{aligned}
& \alpha_{k, \text { min }} \triangleq \frac{\gamma_{1} \sigma_{D_{1}}^{2}}{P_{R_{k}} g_{R_{k} D_{1}}}, \\
& \alpha_{k, \text { max }} \triangleq \min \left\{\frac{P_{R_{k}} g_{R_{k} D_{1}}-\gamma_{2} \sigma_{D_{1}}^{2}}{P_{R_{k}} g_{R_{k} D_{1}}\left(1+\gamma_{2}\right)}, \frac{P_{R_{k}} g_{R_{k} D_{2}}-\gamma_{2} \sigma_{D_{2}}^{2}}{P_{R_{k}} g_{R_{k} D_{2}}\left(1+\gamma_{2}\right)}\right\} .
\end{aligned}
$$

Likewise, for decoding $x_{1}$ first at both destinations, we have:

$$
\begin{aligned}
& \alpha_{k, \text { min }} \triangleq \max \left\{\frac{\gamma_{1}\left(P_{R_{k}} g_{R_{k} D_{1}}+\sigma_{D_{1}}^{2}\right)}{P_{R_{k}} g_{R_{k} D_{1}}\left(1+\gamma_{1}\right)}, \frac{\gamma_{1}\left(P_{R_{k}} g_{R_{k} D_{2}}+\sigma_{D_{2}}^{2}\right)}{P_{R_{k}} g_{R_{k} D_{2}}\left(1+\gamma_{1}\right)}\right\}, \\
& \alpha_{k, \text { max }} \triangleq \frac{P_{R_{k}} g_{R_{k} D_{2}}-\gamma_{2} \sigma_{D_{2}}^{2}}{P_{R_{k}} g_{R_{k} D_{2}}} .
\end{aligned}
$$

Let vector $b_{R D} \triangleq\left(b_{R_{1} D}, b_{R_{2} D}, \ldots, b_{R_{K} D}\right)$ be the binary representation of the $\{R \rightarrow D\}$ links satisfying Equations (6) and (7) and so, if a NOMA transmission on the set of links $\left\{R_{k} \rightarrow D_{1}\right\}$, $\left\{R_{k} \rightarrow D_{2}\right\}$ is possible, then $b_{R_{k} D}=1$. Correspondingly, let vector $q_{R D} \triangleq\left(q_{R_{1} D}, q_{R_{2} D}, \ldots, q_{R_{K} D}\right)$ be the binary representation of the feasible links due to the fulfillment of the buffer conditions, i.e., $\{R \rightarrow D\}$ links where relays have non-empty buffers. By $\mathcal{F}_{R D}$, we denote the sets $\{R \rightarrow D\}$ links that are feasible with cardinality $F_{R D}$.

\subsection{Transmission in the $\{S \rightarrow R\}$ Link}

In this topology, a time slot is dedicated to concurrent $\{S \rightarrow R\}$ and $\{R \rightarrow D\}$ transmissions. As a result, if a transmitting relay $R_{k}$ has been selected, the reception of the other $K-1$ relays will experience the degrading effect of IRI. Regarding the source broadcast signal, as each destination might demand a different rate $r_{j}, j \in\{1,2\}$, and in order to avoid instances of buffer overflowing or underflowing, the source transmits with rate $r_{1}+r_{2}$ [29]. Therefore link $S R_{i}, i \neq k$ is not in outage when:

$$
\Gamma_{S R_{i}}\left(P_{S}\right) \triangleq \frac{g_{S R_{i}} P_{S}}{g_{R_{k} R_{i}} P_{R_{k}}+\sigma_{i}^{2}} \geq 2^{r_{1}+r_{2}}-1 .
$$

On the contrary, link $S R_{i}$ is in outage if $\gamma_{R_{i}}<2^{r_{1}+r_{2}}-1$, and its outage probability is given by:

$$
p_{\text {out }\{S \rightarrow R\}} \triangleq \mathbb{P}\left[g_{S R_{i}}<\frac{\left(2^{r_{1}+r_{2}}-1\right)\left(g_{R_{k} R_{i}} P_{R_{k}}+\sigma_{i}^{2}\right)}{P_{S}}\right] .
$$


The vector $b_{S R} \triangleq\left(b_{S R_{1}}, b_{S R_{2}}, \ldots, b_{S R_{K}}\right)$ with binary elements includes the $\{S \rightarrow R\}$ links that are not in outage. So, if transmission on link $S R_{i}$ is feasible, then $b_{S R_{i}}=1$. Similarly, $q_{S R} \triangleq$ $\left(q_{S R_{1}}, q_{S R_{2}}, \ldots, q_{S R_{K}}\right)$ represents in a binary form, the feasible $\{S \rightarrow R\}$ links due to the fulfillment of the queue conditions. More specifically, buffer conditions are not violated in case of non-full buffers in the $\{S \rightarrow R\}$ links. Set $\mathcal{F}_{S R}$ comprises the feasible $\{S \rightarrow R\}$ links. If $b_{S R_{i}}=0$ or $q_{S R_{i}}=0$, the reception of the source broadcast signal is not possible on link $S R_{i}$ and thus it is considered to be in outage.

\subsection{Discussion and Summary}

Regarding the practical implementation of BASSA-NOMA, each relay $R_{k}$ with packets in its buffer must compute the values $\alpha_{k \text {,min }}$ and $\alpha_{k, \max }$, examining if a NOMA transmission is feasible. Considering the availability of $\{R \rightarrow D\}$ CSI at each $R_{k}$, the range of the $\alpha_{k}$ values can be precisely derived. After this process has been completed, if $\mathcal{F}_{R D} \neq \varnothing, R_{k} \in \mathcal{F}_{R D}$ is chosen as the transmitting relay, having the maximum number of packets in its buffer. In order to avoid relay selection with outdated CSI, as a result of centralized CSI acquisition and processing, distributed solutions should be prioritized [14]. A fully distributed method for network coordination relying on BSI has been presented in [9], based on the use of timers at the relays, setting their values to be inversely proportional to the buffer size of each relay belonging to $\mathcal{F}_{R D}$.

In addition, the use of $\{S \rightarrow R\}$ broadcasting might introduce duplicate packets among the $K$ relays. As a countermeasure to avoid the possibility of duplicate packets, the two destinations are employed to broadcast ACKs, including the identity of the successfully received packets, thus enabling the relays, storing the same packets in their buffers to discard them. If $\mathcal{F}_{R D}=\varnothing$, no relay exists to be activated for transmission and the source broadcast transmission is performed without any IRI degrading the reception of the relays.

Furthermore, the power allocation process in NOMA requires the order with which, the packets will be decoded at each destination. Thus, the selected transmitting relay $R_{k}$ must notify both destinations on the decoding strategy that they must follow. This process can be performed with an additional bit to the packet's header. In case the bit value is " 0 ", $D_{1}$ will be directed to adopt SIC and decode its packet interference-free after decoding and subtracting the packet of $D_{2}$. Simultaneously, $D_{2}$ will attempt to decode its packet by treating the signal of $D_{1}$, as interference. The reverse strategies are followed when the bit value is " 1 ". It must be noted that each candidate transmitting relay $R_{k}$ evaluates all the possible decoding strategies and when the first one satisfying the rate requirements is identified, the decoding strategy is broadcasted to $D_{1}$ and $D_{2}$.

\section{Performance Evaluation}

Following the description of the system model in Section 2, BASSA-NOMA's performance is evaluated for different network parameters, in terms of outage probability, average sum-rate and average delay for the transmitted packets. In the simulations, the noise level is assumed to be equal to $1 \mathrm{~mW}$ while the wireless channels are independent non identically distributed (i.n.i.d). Moreover, two destinations exist, requiring equal rates $r_{1}=r_{2}=r_{D}=2 \mathrm{bps} / \mathrm{Hz}$ and their channel asymmetry is defined as $\sigma_{S R_{k}}^{2}=\sigma_{R_{k} D_{1}}^{2}=4 \sigma_{R_{k} D_{2}}^{2}$. In addition, BASSA-NOMA is evaluated under different channel conditions, in terms of line-of-sight (LoS). When LoS conditions occur, the Rician factor $K_{\text {Rice }}$ is assumed to be equal to $10 \mathrm{~dB}$.

In the following paragraphs, comparisons are presented among BASSA-NOMA and other NOMA and OMA schemes. In order to ensure fairness while comparing NOMA and OMA algorithms, the OMA transmission in each hop is performed with twice the rate, as for end-to-end communication, twice the time slots of NOMA are required, with $D_{1}$ receiving in odd time slots and $D_{2}$ in even time slots. Moreover, outages in OMA occur if at two consecutive time slots, all the relays fail to successfully transmit/receive a packet, while in NOMA, outages are experienced if at an arbitrary time slot, no relay exists to perform a successful transmission/reception. 


\subsection{Comparisons with Other Schemes}

Here, comparisons include the OMA schemes of [14] (LoCo - Link) and [39] (OMA RD), as well as equivalent NOMA-based versions (LoCo - Link-NOMA and NOMA RD). Finally, the hybrid BA-HD-NOMA/OMA of [31] is compared, where if NOMA fails, a single user $D_{i}$ is served with rate $r_{i}, i \in\{1,2\}$. However, BA-HD-NOMA/OMA is excluded from the outage results, as it cannot be directly compared to standalone NOMA and OMA [29].

\subsubsection{Outage Probability}

The first comparison focuses on the outage probability performance, in a network with $K=3$ relays and $L=8$. The results are included in Figure 2. Firstly, it can be seen that among the HD schemes, the NOMA-based versions provide improved outage probability compared to their OMA equivalents, as optimal power allocation is performed, exploiting the availability of $\{R \rightarrow D\}$ CSI. Moreover, for high transmit SNR values, BASSA-NOMA outperforms the outage performance of both HD NOMA schemes. This behaviour stems from the successive $\{S \rightarrow R\}$ and $\{R \rightarrow D\}$ transmissions at each time slot, as SuR allows more packets to be distributed at the relays due to source broadcasting, while instances of full buffers and reduced diversity are avoided, as a result of the transmitting relay's NOMA transmissions at each time slot.

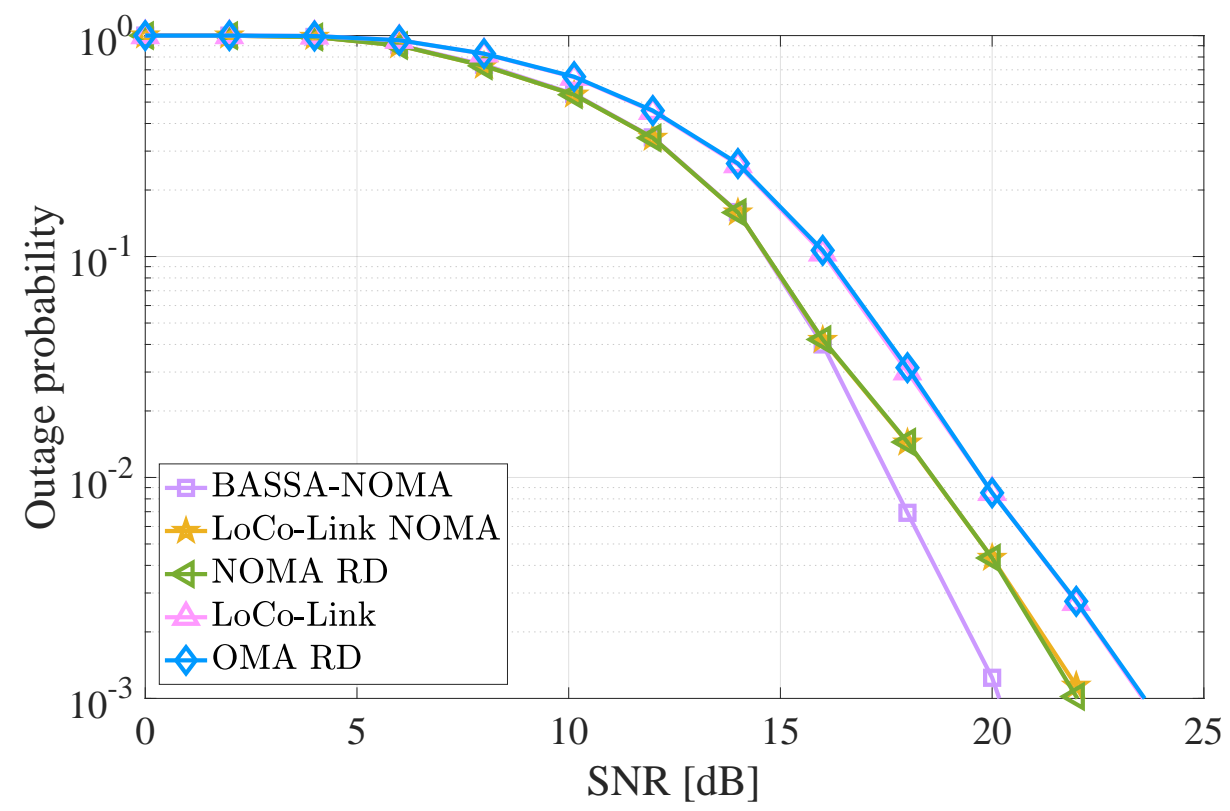

Figure 2. Outage probability for $K=3, L=8$ and various algorithms.

\subsubsection{Average Sum-Rate}

Regarding the average sum-rate performance, Figure 3 presents the results for different relay selection schemes, in a network with $K=3$ relays and $L=8$. For low transmit SNR, BA-HD-NOMA/OMA provides the best performance, as it can maintain a higher sum-rate by switching to OMA when NOMA power allocation is insufficient to improve the SIC performance. However, after $12 \mathrm{~dB}$, BASSA-NOMA provides clear gains, as it is the only scheme that can achieve FD transmissions, capitalizing on the SuR principle, even if it introduces IRI to the network. More importantly, the source's broadcast transmissions, enabling $K-1$ relays to attempt a reception improves the chances of successful $\{S \rightarrow R\}$ communication, without requiring CSI at the source, thus entailing reduced coordination overheads. 


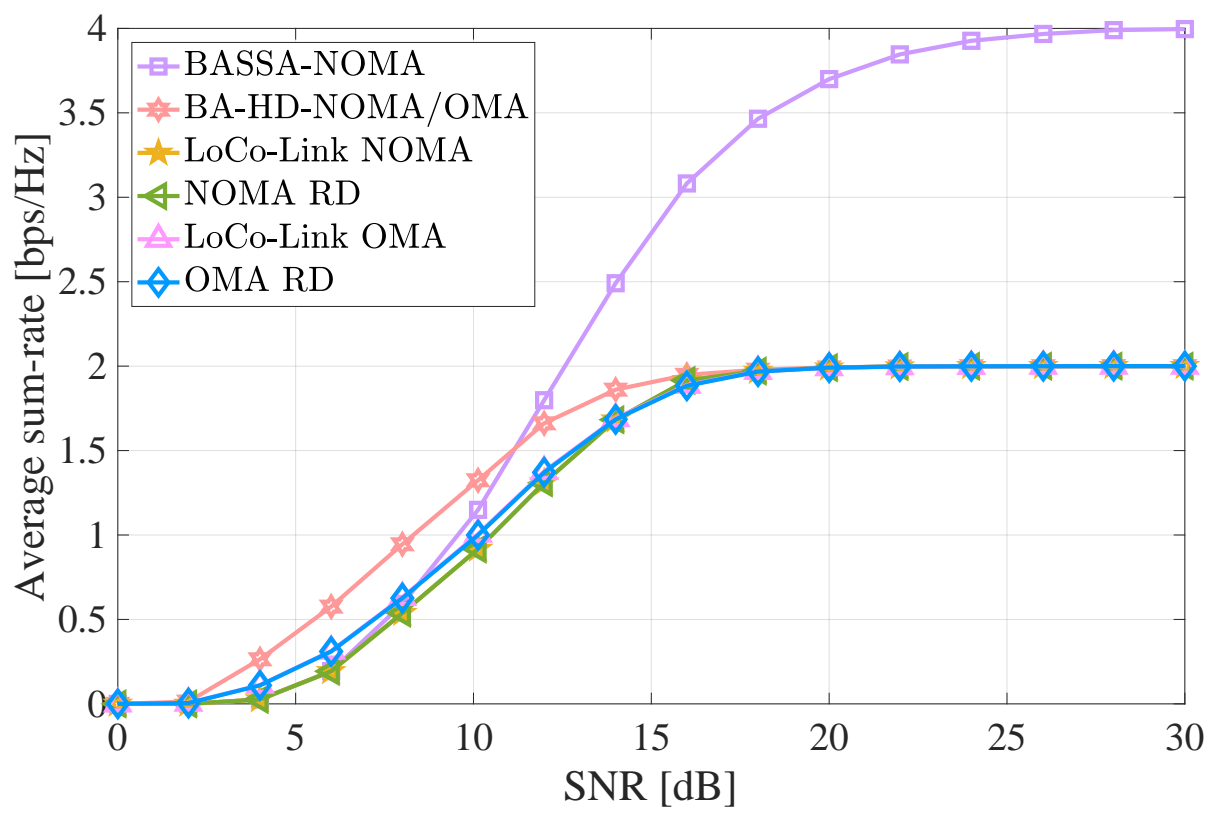

Figure 3. Average sum-rate for $K=3, L=8$ and various algorithms.

Next, the effect of increasing the number of available relays $K$ is evaluated in Figure 4 . It is evident that by increasing $K$, the effect of IRI in the network can be mitigated, as with each additional relay, the available degrees of freedom are increased. Moreover, it can be seen that having the minimum number of relays for SuR, i.e., $K=2$ results in significantly worse performance, compared to $K=3$ and $K=4$. In this case, the network is much more vulnerable to both IRI and instances of reduced diversity when buffers are empty or full.

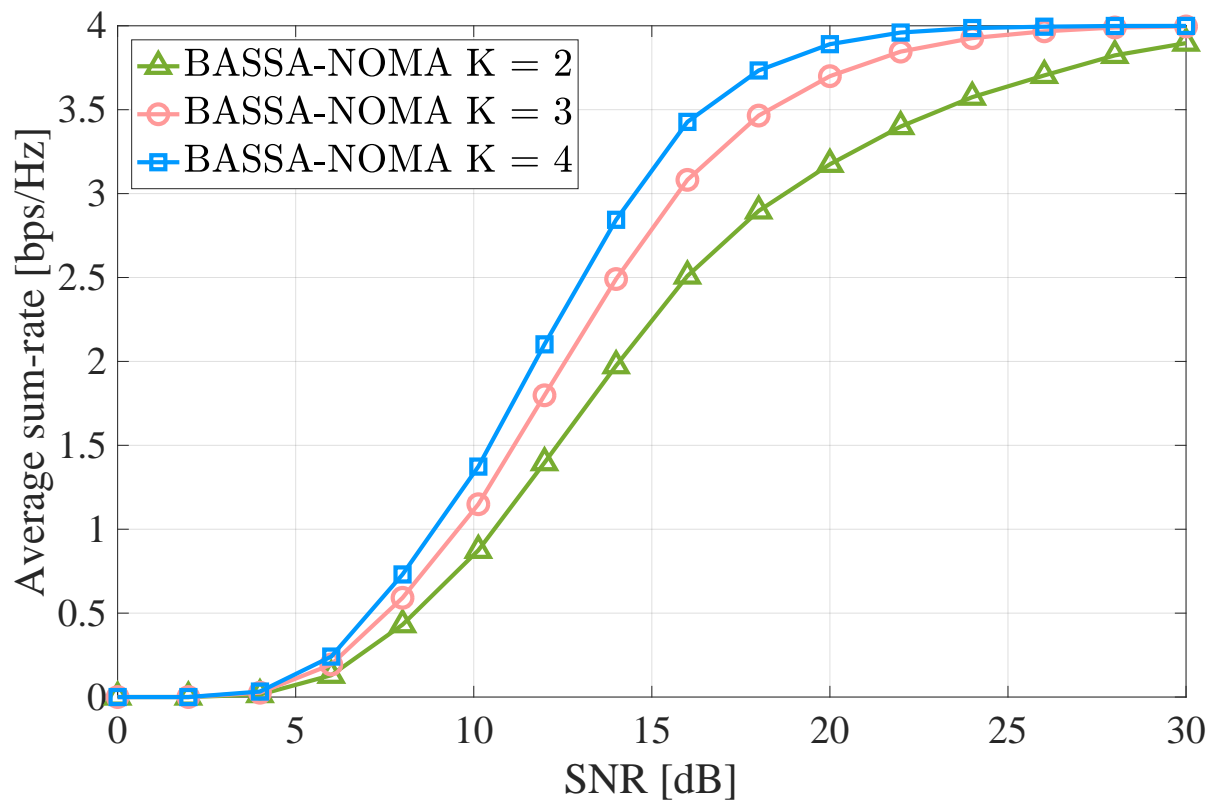

Figure 4. Average sum-rate for BASSA-NOMA (buffer-aided and successive single-antenna non-orthogonal multiple access)for varying $K$ and $L=8$.

Another sum-rate comparison is given in Figure 5. Here, $K=3$ relays and $L=8$ and each time different rate requirements are considered. From the results, it can be observed that as the transmit SNR increases, the sum-rate that can be supported by the network increases as well. Thus, it should be noted that, in highly dynamic topologies where the average SNR conditions abruptly change, 
there is significant potential in adopting BASSA-NOMA and adjusting its operation for adaptive rate transmissions by the source and the transmitting relay.

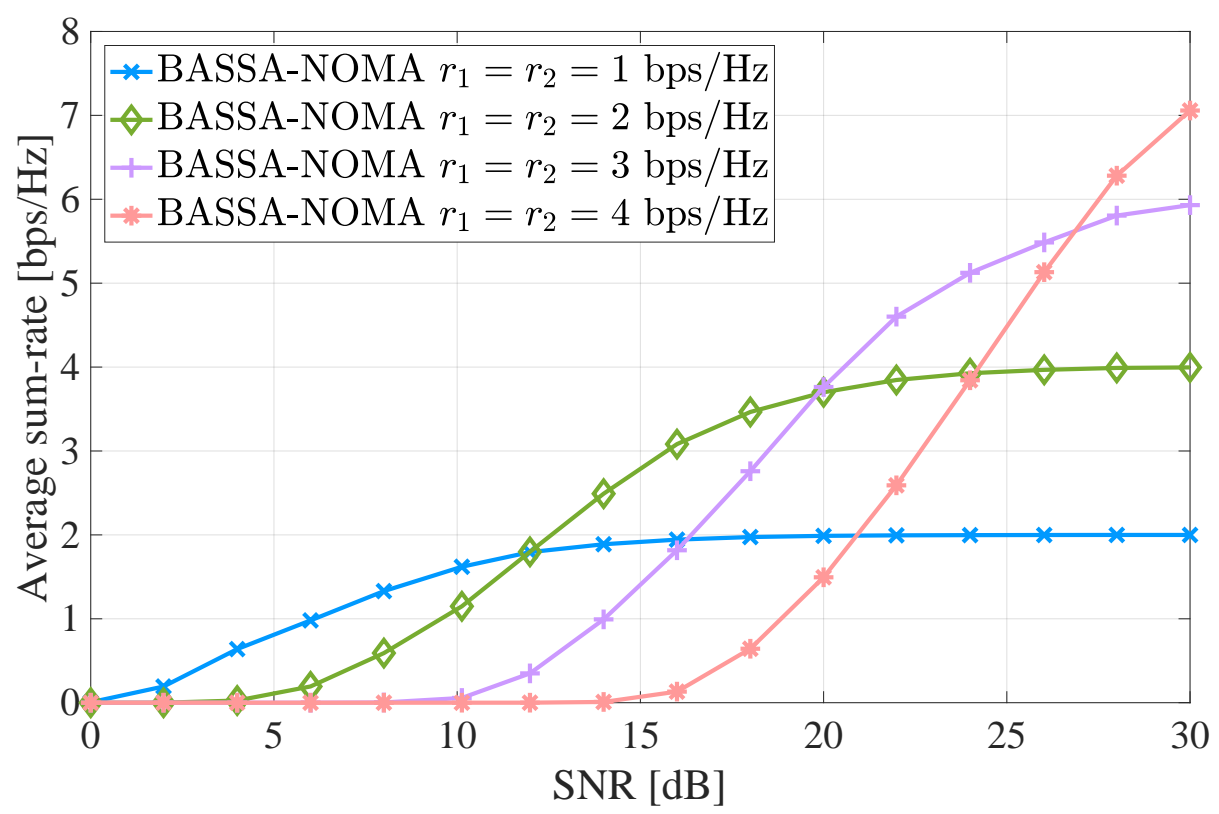

Figure 5. Average sum-rate for BASSA-NOMA for $K=3, L=8$ and varying rate requirements.

\subsubsection{Average Delay}

In BA relay networks, the average delay performance is of high importance, as inefficient selection schemes might lead to packets residing in the buffers for a large number of time slots. So, the final comparison in Figure 6 deals with the average delay performance of the transmitted packets for various selection schemes. In this comparison, it can be seen that all the schemes converge to an average delay of one time slot. This is the result of integrating DA mechanisms in every scheme. Nonetheless, as it was shown in the sum-rate comparisons, BASSA-NOMA transmits twice the number of packets for high transmit SNR and thus the average delay performance corresponds to an increased number of transmitted packets, compared to the HD schemes.

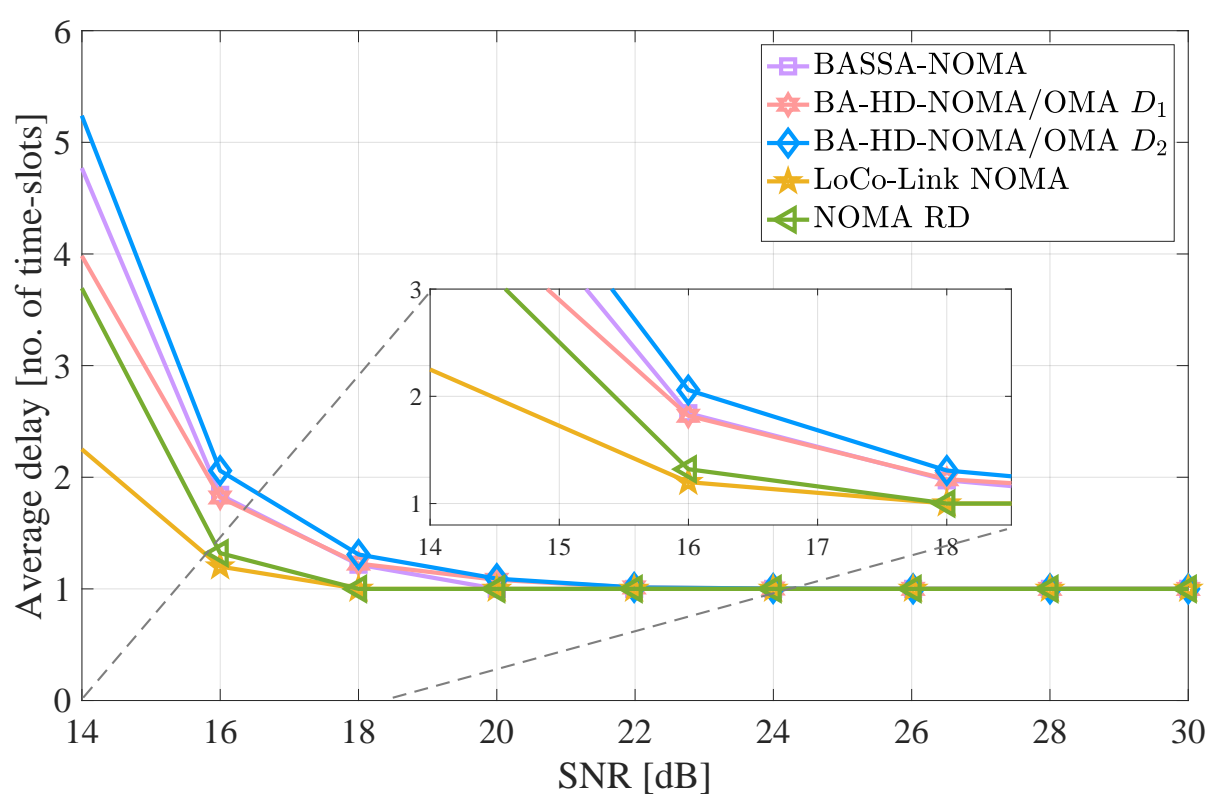

Figure 6. Average delay for $K=3, L=8$ and various algorithms. 


\subsection{Evaluation for Varying Channel Conditions}

Departing from comparing different relay selection schemes under Rayleigh fading, we now focus on evaluating BASSA-NOMA for different wireless channel conditions, in terms of LoS connectivity in each hop.

\subsubsection{Outage Probability}

In Figure 7, four different wireless settings are compared, depending on the LoS conditions in each hop. It can be seen that when LoS conditions occur in the $\left\{R \rightarrow D_{1}\right\}$ link while the $\{S \rightarrow R\}$ links experience NLoS channels, for low and medium SNR, improved performance is observed as the chances for successful NOMA increase due to the channel asymmetry, allowing more efficient power allocation to be performed. Still, as SNR increases beyond $18 \mathrm{~dB}$ and thus NOMA can be efficiently performed without LoS conditions, the same performance is experienced as in the case of Rayleigh fading in both hops. On the contrary, for the case of LoS conditions only in the $\{S \rightarrow R\}$, improved diversity is observed after $18 \mathrm{~dB}$. Overall, the best performance is exhibited in the topology where LoS and Rice fading exist in both the $\{S \rightarrow R\}$ and $\left\{R \rightarrow D_{1}\right\}$ links, limiting the instances of unsuccessful reception by the relays in the first hop and increasing the chances for successful NOMA transmission to both destinations.

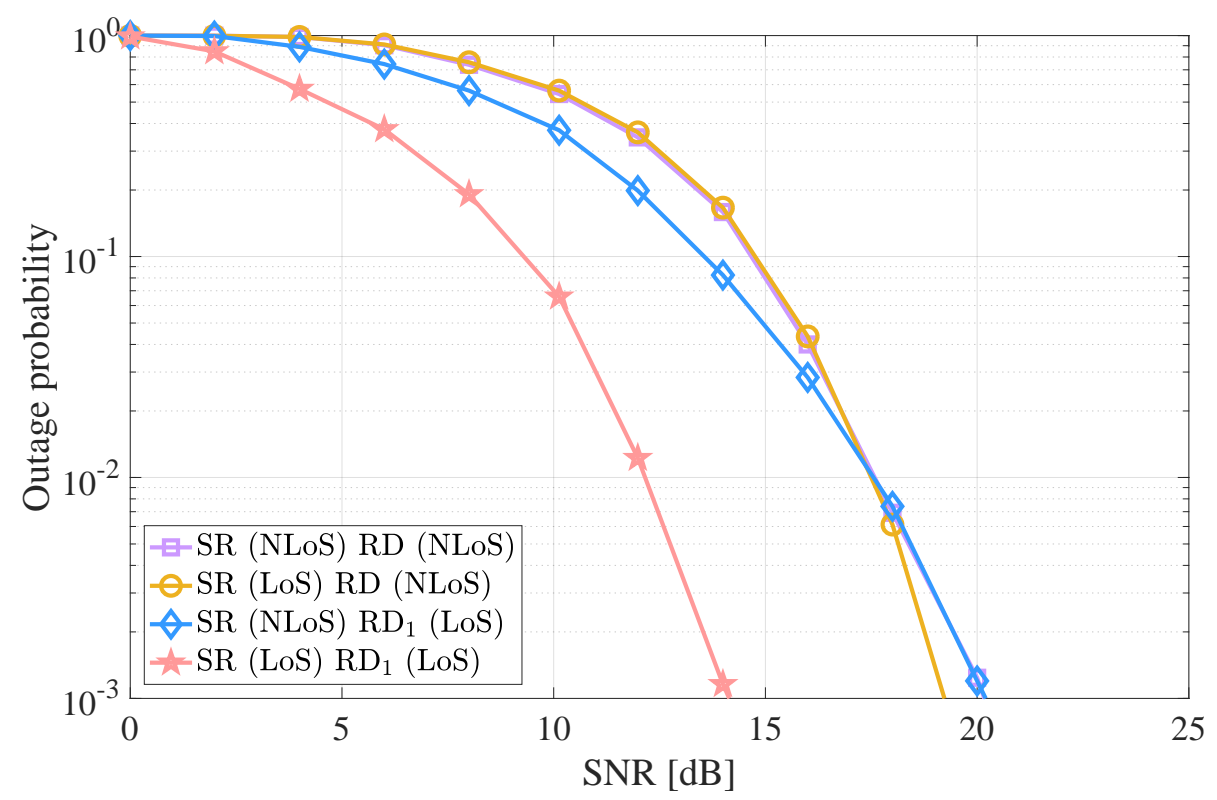

Figure 7. Outage probability for $K=3, L=8$ and varying channel conditions.

\subsubsection{Average Sum-Rate}

Then, Figure 8 depicts the average sum-rate performance of the four topologies where BASSA-NOMA is adopted. It can be seen that the case of Rayleigh fading in both hops provides the worst performance, as in the low and medium SNR, NOMA cannot be efficiently performed, while the source's broadcast transmissions reaches a diversity threshold in the fow SNR regime. The mixed cases of Rice and Rayleigh fading follow their respective outage probability performance, each offering improved performance for low to medium SNR $\left(\{S \rightarrow R\}\right.$ with Rayleigh and $\left\{R \rightarrow D_{1}\right\}$ with Rice) and high SNR ( $\{S \rightarrow R\}$ with Rice and $\left\{R \rightarrow D_{1}\right\}$ with Rayleigh). Again, the best sum-rate results are achieved in the topology with Rice fading in both the $\{S \rightarrow R\}$ and $\left\{R \rightarrow D_{1}\right\}$ links, thus highlighting the importance of using relays, having optimized positions in relation to the source and the destinations. This behavior strengthens the importance of using mobile relays in wireless networks, where dynamic repositioning can be performed, exploiting the CSI at the relays. 


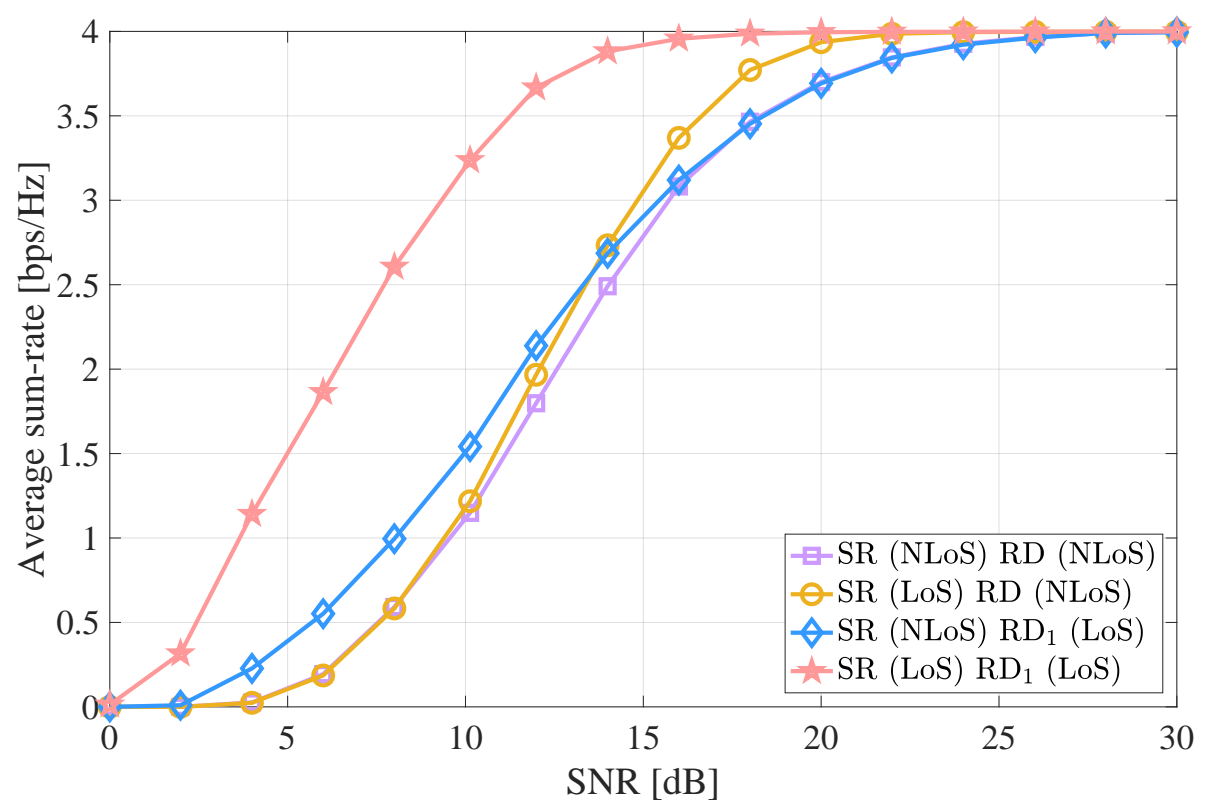

Figure 8. Average sum-rate for $K=3, L=8$ and varying channel conditions.

\section{Conclusions and Future Directions}

The ever-increasing desire of users to enjoy highly demanding services, in terms of rate and delay has put excessive stress on wireless networks. At the same time, recent breakthroughs have lead to the development of novel transmission techniques, improving the performance of wireless deployments. Moreover, the heterogeneity of wireless networks, comprising nodes with different hardware and processing capabilities cannot be ignored and thus efficient algorithms must be devised, offering high performance with low-complexity implementation, exploiting apart from fixed infrastructure-based relays, users and mobile relay nodes. This work focused on leveraging the capabilities provided by low-complexity single-antenna cooperative relays with buffers and non-orthogonal multiple access. More specifically, an opportunistic relay selection algorithm was developed, allowing a cluster of single-antenna relays to operate in full-duplex mode through the successive relaying principle. In addition, buffering facilitated the seamless combination of half-duplex relaying with successive source broadcasting and relay transmissions, enhancing the reliability of the two-hop NOMA relay network, and lowering the delay of the transmission, while increasing the number of transmitted packets to both destinations.

The findings in this study provide further opportunities to investigate the applicability of BASSA-NOMA in different scenarios. An important process towards improving the performance of NOMA networks is to form user clusters, in order to exploit channel asymmetries and different rate requirements. So, the integration of efficient clustering algorithms [40], improving the chances for SIC and user fairness, is of high importance. Moreover, in this work, we focused on a two-user topology and leveraged efficient power allocation for improved NOMA performance. Nonetheless, aiming to support massive connectivity requirements of $5 \mathrm{G}$ and beyond networks, the generalization of the BASSA-NOMA operation to topologies with arbitrary numbers of users is necessary. Moreover, the advantage of BASSA-NOMA, in terms of reliability can be beneficial to aerial networks where connectivity conditions are subject to abrupt changes. Finally, adjusting the operation of BASSA-NOMA with adaptive rate transmissions represents another interesting direction to further improve the performance of NOMA relay networks.

Author Contributions: Conceptualization, N.N.; methodology, N.N. and P.T.; validation, P.T., A.H. and S.V.; formal analysis, N.N. and P.T.; investigation, N.N., P.T. and A.H.; writing-original draft preparation, N.N. and P.T.; writing-review and editing, A.H. and S.V. 
Funding: This research received no external funding.

Conflicts of Interest: The authors declare no conflict of interest.

\section{Abbreviations}

The following abbreviations are used in this manuscript:

5G Fifth Generation

ACK Acknowledgment

AWGN Additive White Gaussian Noise

BA Buffer-Aided

BASSA Buffer-Aided Successive Single-Antenna

BSI Buffer State Information

CD Code Domain

CSI Channel State Information

DA Delay-Aware

DDA Delay- and Diversity-Aware

DF Decode-and-Forward

FD Full-Duplex

HD Half-Duplex

HRS Hybrid Relay Selection

IoT Internet-of-Things

IRI Inter-Relay Interference

LoCo Low-Complexity

LoLA4SOR Low-Latency for Successive Opportunistic Relaying

LoS Line-of-Sight

MMRS Max-Max Relay Selection

NACK Negative-Acknowledgment

NLoS Non-Line-of-Sight

NOMA Non-Orthogonal Multiple Access

OMA Orthogonal Multiple Access

ORS Opportunistic Relay Selection

PD Power Domain

RD Relay-Destination

SD Source-Destination

SFD Space Full-Duplex

SIC Successive Interference Cancellation

SINR Signal-to-Interference-plus-Noise Ratio

SNR Signal-to-Noise Ratio

SR Source-Relay

SuR Successive Relaying

\section{References}

1. Ericsson Mobility Report. Available online: https://www.ericsson.com/assets/local/mobility-report/ documents/2019/ericsson-mobility-report-june-2019.pdf (accessed on 11 November 2019).

2. Ding, Z.; Liu, Y.; Choi, J.; Sun, Q.; Elkashlann, M.; Chih-Lin, I.; Poor, H.V. Application of Non-Orthogonal Multiple Access in LTE and 5G Networks. IEEE Commun. Mag. 2017, 55, 185-191. [CrossRef]

3. Dai, L.; Wang, B.; Yuan, Y.; Han, S.; Chih-Lin, I.; Wang, Z. Non-Orthogonal Multiple Access for 5G: Solutions, Challenges, Opportunities, and Future Research Trends. IEEE Commun. Mag. 2015, 53, 74-81. [CrossRef]

4. Islam, S.M.R.; Avazov, N.; Dobre, O.A.; Kwak, K.S. Power-Domain Non-Orthogonal Multiple Access (NOMA) in 5G Systems: Potentials and Challenges. IEEE Commun. Surv. Tutor. 2017, 19, 721-742. [CrossRef]

5. Ding, Z.; Lei, X.; Karagiannidis, G.K.; Schober, R.; Yuan, J.; Bhargava, V.K. A Survey on Non-Orthogonal Multiple Access for 5G Networks: Research Challenges and Future Trends. IEEE J. Sel. Areas Commun. 2017, 35, 2181-2195. [CrossRef] 
6. Zlatanov, N.; Ikhlef, A.; Islam, T.; Schober, R. Buffer-Aided Cooperative Communications: Opportunities and Challenges. IEEE Commun. Mag. 2014, 52, 146-153. [CrossRef]

7. Nomikos, N.; Charalambous, T.; Krikidis, I.; Skoutas, D.N.; Vouyioukas, D.; Johansson, M.; Skianis, C. A Survey on Buffer-Aided Relay Selection. IEEE Commun. Surv. Tutor. 2016, 18, 1073-1097. [CrossRef]

8. Qiao, D.Q.; Gursoy, M.C. Buffer-Aided Relay Systems Under Delay Constraints: Potentials and Challenges. IEEE Commun. Mag. 2017, 55, 168-174. [CrossRef]

9. Nomikos, N.; Poulimeneas, D.; Charalambous, T.; Krikidis, I.; Vouyioukas, D.; Johansson, M. Delay- and Diversity-Aware Buffer-Aided Relay Selection Policies in Cooperative Networks. IEEE Access 2018, 6, 73531-73547. [CrossRef]

10. Ikhlef, A.; Michalopoulos, D.S.; Schober, R. Max-Max Relay Selection for Relays with Buffers. IEEE Trans. Wirel. Commun. 2012, 11, 1124-1135. [CrossRef]

11. Krikidis, I.; Charalambous, T.; Thompson, J.S. Buffer-Aided Relay Selection for Cooperative Diversity Systems without Delay Constraints. IEEE Trans. Wirel. Commun. 2012, 11, 1957-1967. [CrossRef]

12. Zlatanov, N.; Schober, R. Buffer-Aided Half-Duplex Relaying Can Outperform Ideal Full-Duplex Relaying. IEEE Commun. Lett. 2013, 17, 479-482. [CrossRef]

13. Oiwa, M.; Sugiura, S. Reduced-Packet-Delay Generalized Buffer-Aided Relaying Protocol: Simultaneous Activation of Multiple Source-to-Relay Links. IEEE Access 2016, 4, 3632-3646. [CrossRef]

14. Nomikos, N.; Charalambous, T.; Vouyioukas, D.; Karagiannidis, G.K. Low-Complexity Buffer-Aided Link Selection with Outdated CSI and Feedback Errors. IEEE Trans. Commun. 2018, 66, 3694-3706. [CrossRef]

15. Ikhlef, A.; Junsu, K.; Schober, R. Mimicking Full-Duplex Relaying Using Half-Duplex Relays with Buffers. IEEE Trans. Veh. Technol. 2012, 61, 3025-3037. [CrossRef]

16. Nomikos, N.; Vouyioukas, D.; Charalambous, T.; Krikidis, I.; Makris, P.; Skoutas, D.N.; Johansson, M.; Skianis, C. Joint Relay-Pair Selection for Buffer-Aided Successive Opportunistic Relaying. Trans. Emerg. Telecommun. Technol. 2014, 25, 823-834. [CrossRef]

17. Nomikos, N.; Charalambous, T.; Krikidis, I.; Skoutas, D.N.; Vouyioukas, D.; Johansson, M. A Buffer-Aided Successive Opportunistic Relaying Selection Scheme with Power Adaptation and Inter-Relay Interference Cancellation for Cooperative Diversity Systems. IEEE Trans. Commun. 2015, 63, 1623-1634. [CrossRef]

18. Nomikos, N.; Charalambous, T.; Pappas, N.; Vouyioukas, D.; Wichman, R. LoLA4SOR: A Low-Latency Algorithm for Successive Opportunistic Relaying. In Proceedings of the IEEE International Conference on Computer Communications: Workshop on Ultra-Low Latency in Wireless Networks (ULLWN), Paris, France, 29 April-2 May 2019.

19. Simoni, R.; Jamali, V.; Zlatanov, N.; Schober, R.; Pierucci, L.; Fantacci, R. Buffer-Aided Diamond Relay Network with Block Fading and Inter-Relay Interference. IEEE Trans. Wirel. Commun. 2016, 15, 7357-7372. [CrossRef]

20. Gupta, S.; Zhang, R.; Hanzo, L. Throughput Maximization for a Buffer-Aided Successive Relaying Network Employing Energy Harvesting. IEEE Trans. Veh. Technol. 2016, 65, 6758-6765. [CrossRef]

21. Shabbir, G.; Ahmad, J.; Raza, W.; Amin, Y.; Akram, A.; Loo, J.; Tenhunen, H. Buffer-Aided Successive Relay Selection Scheme for Energy Harvesting IoT Networks. IEEE Access 2019, 7, 36246-36258. [CrossRef]

22. Ding, Z.; Peng, M.; Poor, H.V. Cooperative Non-Orthogonal Multiple Access in 5G Systems. IEEE Commun. Lett. 2015, 19, 1462-1465. [CrossRef]

23. Ding, Z.; Dai, H.; Poor, H.V. Relay Selection for Cooperative NOMA. IEEE Wirel. Commun. Lett. 2016, 5, 416-419. [CrossRef]

24. Zhang, Y.; Wang, X.; Wang, D.; Zhao, Q.; Zhang, Y. A Range-Division User Relay Selection Scheme and Performance Analysis in NOMA-based Cooperative Opportunistic Multicast Systems. Electronics 2019, 8, 544. [CrossRef]

25. Liau, Q.Y.; Leow, C.Y.; Ding, Z. Amplify-and-Forward Virtual Full-Duplex Relaying-Based Cooperative NOMA. IEEE Wirel. Commun. Lett. 2018, 7, 464-467. [CrossRef]

26. Wang, S.; Cao, S.; Ruby, R. Optimal Power Allocation in NOMA-Based Two-Path Successive AF Relay Systems. EURASIP J. Wirel. Commun. Netw. 2018, 2018, 273. [CrossRef]

27. Liau, Q.Y.; Leow, C.Y. Successive User Relaying in Cooperative NOMA System. IEEE Wirel. Commun. Lett. 2019, 8, 921-924. [CrossRef]

28. Luo, S.; Teh, K.C. Adaptive Transmission for Cooperative NOMA System with Buffer-Aided Relaying. IEEE Commun. Lett. 2017, 21, 937-940. [CrossRef] 
29. Zhang, Q.; Liang, Z.; Li, Q.; Qin, J. Buffer-Aided Non-Orthogonal Multiple Access Relaying Systems in Rayleigh Fading Channels. IEEE Trans. Commun. 2017, 65, 95-106. [CrossRef]

30. Nomikos, N.; Charalambous, T.; Vouyioukas, D.; Karagiannidis, G.K.; Wichman, R. Relay Selection for Buffer-Aided Non-Orthogonal Multiple Access Networks. In Proceedings of the IEEE GLOBECOM: Workshop on Non-Orthogonal Multiple Access Techniques for 5G, Singapore, 4-8 December 2017.

31. Nomikos, N.; Charalambous, T.; Vouyioukas, D.; Karagiannidis, G.K.; Wichman, R. Hybrid NOMA/OMA with Buffer-Aided Relay Selection in Cooperative Networks. IEEE J. Sel. Top. Signal Process. 2019, 13, 524-537. [CrossRef]

32. Nomikos, N.; Michailidis, E.T.; Trakadas, P.; Vouyioukas, D.; Zahariadis, T.; Krikidis, I. Flex-NOMA: Exploiting Buffer-Aided Relay Selection for Massive Connectivity in the 5G Uplink. IEEE Access 2019, 7, 88743-88755. [CrossRef]

33. Dun, H.; Ye, F.; Jiao, S.; Liu, D. Power Control for Device-to-Device Communication with a Hybrid Relay Mode in Unequal Transmission Slots. Electronics 2018, 7, 17. [CrossRef]

34. Khan, U.A.; Lee, S.S. Three-Dimensional Resource Allocation in D2D-Based V2V Communication. Electronics 2019, 8, 962. [CrossRef]

35. Michailidis, E.T.; Nomikos, N.; Bithas, P.S.; Vouyioukas, D.; Kanatas, A.G. Optimal 3-D Aerial Relay Placement for Multi-User MIMO Communications. IEEE Trans. Aerosp. Electron. Syst. 2019. [CrossRef]

36. Singh, K.; Gupta, A.; Ratnarajah, T.; Ku, M. A General Approach Toward Green Resource Allocation in Relay-Assisted Multiuser Communication Networks. IEEE Trans. Wirel. Commun. 2018, 17, 848-862. [CrossRef]

37. Singh, K.; Gupta, A.; Ratnarajah, T. A Utility-Based Joint Subcarrier and Power Allocation for Green Communications in Multi-User Two-Way Regenerative Relay Networks. IEEE Trans. Commun. 2017, 65, 3705-3722. [CrossRef]

38. Krikidis, I.; Thompson, J.; McLaughlin, S.; Goertz, N. Amplify-and-Forward with Partial Relay Selection. IEEE Commun. Lett. 2008, 12, 235-237. [CrossRef]

39. Tian, Z.; Gong, Y.; Chen, G.; Chambers, J.A. Buffer-Aided Relay Selection with Reduced Packet Delay in Cooperative Networks. IEEE Trans. Veh. Technol. 2017, 66, 2567-2575. [CrossRef]

40. Singh, K.; Wang, K.; Biswas, S.; Ding, Z.; Khan, F.A.; Ratnarajah, T. Resource Optimization in Full Duplex Non-Orthogonal Multiple Access Systems. IEEE Trans. Wirel. Commun. 2019, 18, 4312-4325. [CrossRef]

(C) 2019 by the authors. Licensee MDPI, Basel, Switzerland. This article is an open access article distributed under the terms and conditions of the Creative Commons Attribution (CC BY) license (http://creativecommons.org/licenses/by/4.0/). 